\title{
Intermittency in the Transition to Turbulence for a Shear-Thinning Fluid in Hagen-Poiseuille Flow
}

\author{
S. A. Bahrani ${ }^{\dagger 1,2}$ and C. Nouar ${ }^{1}$ \\ 1 LEMTA, UMR 7563 CNRS, University of Lorraine, 54504 Vandoeuvre-les-Nancy, France \\ 2 IRTES-SeT, University of Technology Belfort-Montbéliard, 90010 Belfort Cedex, France \\ $\dagger$ Corresponding Author Email: seyed-amir.bahrani@univ-lorraine.fr
}

(Received September 20, 2012; accepted February 25, 2013)

\begin{abstract}
Since Reynolds's original experiments in 1883, very few studies have been carried out on the transition to turbulence for shear thinning fluid in Hagen-Poiseuille (pipe) flow. Compared to the Newtonian fluids, the results showed two interesting phenomenon: a delay in transition to turbulence and the appearance of an asymmetry in the mean axial velocity profiles. In this study, we measured experimentally the friction factor and axial velocity profiles using Laser Doppler Velocimetry system (LDV) at different Reynolds numbers. It is shown an axisymmetric in the mean axial velocity profiles for laminar and turbulent regimes and an increasing asymmetry profile with increasing $R e_{w}$ for transition regime. Two different stages are clearly identified during transition to turbulence. The first stage which we called it pre-transition, took place only in the non Newtonian fluids while the second stage correspond to the appearance of turbulent puffs. Another objective of this study is the statistical analysis of the axial velocity fluctuation. In the transition regime, power spectra identified the existence of a weak turbulence. It is generated by the shear-thinning character of the fluid, which follows a power law with an exponent close to $-3\left(f^{-3}\right)$.
\end{abstract}

Keywords: Turbulent transition, Shear thinning fluid, Hagen-Poiseuille flow, Flow instability and density energy spectra

\section{INTRODUCTION}

The mechanism of transition from laminar to turbulence for shear thinning fluid in Hagen-Poiseuille flow through a cylindrical pipe is still unrecognized despite the importance of this problem in the design and control in several industrial process such as cementation in the upstream oil and gas industries, extrusion of molten polymers, paper coating and etc. The main difficultly of this scenario lies on the linear stability of Hagen-Poiseuille flow Meseguer and Trefethen (2003).

Concerning the transitional regime for nonNewtonian fluid flows, the existing literature reveals two interesting phenomenon with yet unexplained effect: a delay in transition to turbulence Escudier et al. (1999) and the appearance of an asymmetry in the mean axial velocity profiles Güzel et al. (2009); Esmael and Nouar (2008); Peixinho et al. (2005).

According to the work of Esmael and Nouar (2008), in the transition regime, a new state with a robust coherent structure characterized by two weakly modulated counter-rotating longitudinal vortices was reported for a yield-stress-thinning fluid in a pipe. In this nonlinear asymmetric state, timeaveraged axial velocity profiles, exhibited increasing asymmetry with increasing Reynolds number. Recently, Escudier et al. (2009) suggested that both upstream and downstream flow geometry have no influence on the symmetry.

Dissipative nonlinear systems such as fluid dynamical systems can reach a chaotic state when the parameter measuring the nonlinearity is large. For instance, parallel shear flows of Newtonian fluids are turbulent when the ratio of the nonlinear inertial term and the viscous dissipative term, defined by the Reynolds number is sufficiently important. In non-Newtonian fluid flows, an additional nonlinearity is introduced via the constitutive equation (Eq. 1). For viscoelastic fluids, this nonlinearity can give rise to a turbulent flow at low Reynolds number Larson (2000); Groisman and Steinberg (2000). 


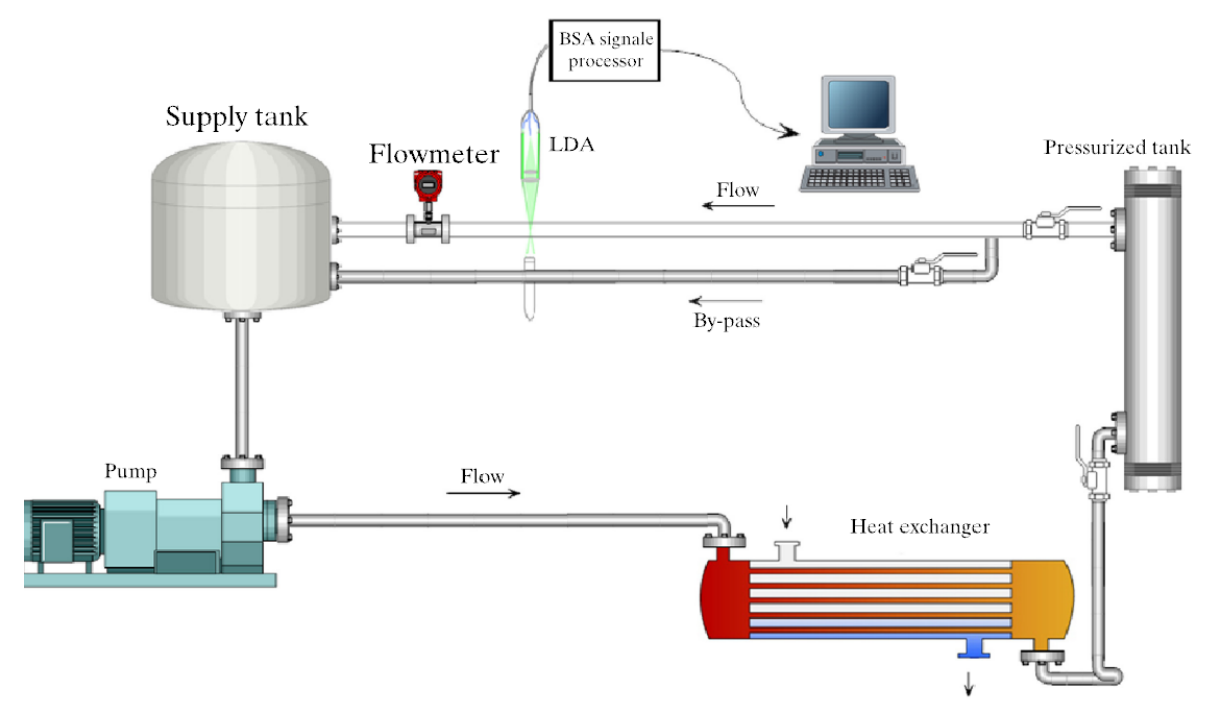

Fig. 1. Schematic of experimental set-up.

$$
\begin{aligned}
& \frac{\partial V}{\partial t}+\overbrace{(V . \nabla) V}^{\text {nonlinear inertial }}=-\frac{1}{\rho} \nabla P+ \\
& \underbrace{\frac{\mu_{s}}{\rho} \Delta V}_{\text {viscous dissipative }}+\frac{1}{\rho} \nabla \tau_{p}
\end{aligned}
$$

Where $\tau_{p}$ is the parietal stress and $\mu_{s}$, the fluid viscosity.

The degree of nonlinearity is expressed by the Weissenberg number which is a product of a characteristic rate of deformation and the relaxation time of the polymer. The shear-thinning behavior, non-linear decrease of the effective viscosity with the shear rate, is the most common property of non-Newtonian fluids. It is reasonable to inquire, whether an interplay between this nonlinearity and inertia can lead to a chaotic flow. This point has been addressed in Ashrafi and Khayat (2000) using low order dynamical system (generalized Lorenz system) in the Taylor-Couette flow of weakly-thinning fluid. It is shown that the additional nonlinearity gives rise to a Hopf bifurcation otherwise non-existent for Newtonian fluid.

In the present paper, we report the first experimental evidence of a weakly turbulent flow induced by the nonlinear dependence of the effective viscosity with the shear rate, resulting from an inter-play between inertia and the shear-thinning behaviour of the fluid. This chaotic flow was discovered in our study of transition to turbulence in a pipe of a non-Newtonian fluid. In this nonlinear state, timeaveraged axial velocity profiles exhibit increasing asymmetry with increasing Reynolds number. A complete statistical analysis of the axial velocity fluctuations is provided with an attempt of interpretation.

\section{EXPERIMENTAL SET-UP AND MEA- SUREMENTS OF FLUID RHEOLOGY}

We carried out the tests to obtain a pressure drop, a "fluctuation and average" axial velocity in the downstream pipe (cylindrical conduit). The experimental set-up (Fig. 1) consists of a Plexiglas pipe with internal diameter of $30 \mathrm{~mm}$ and $4.20 \mathrm{~m}$ long, which is longer than the entry length necessary for the laminar flow. The velocity measurements can be performed by Laser Doppler Velocimetry (LDV, Dantec, Denmark) at horizontal (azimuthal) position.

The applicable fluid is a $0.2 \mathrm{wt} \%$ aqueous solution of carbopol 940, which its stability and transparency has been widely used as a model yield stress. Flow behavior was characterized using the cone/plate geometry $\left(6 \mathrm{~cm} / 1^{\circ}\right)$ and all measurements were carried out in a controlled torque rheometer (TA Instrument AR2000). The HerschelBulkley model was found to satisfactory fit in the flow curves.

\section{Results AND Discussions}

The mean axial velocity profiles shown in Fig. 2a, cover a range of Reynolds numbers which span the three flow regimes at the azimuthal positions of $\theta=$ 0 and the downstream of the pipe. The definition of the Reynolds number is as follows:

$R e_{w}=\frac{\rho U_{B} D}{\mu_{s}}$

Where the viscosity $\mu_{s}$ was determined from the Herschel-Bulkley model. The results show a zone called plug region, in which the rate of strain tensor is zero and $\tau<\tau_{0}$. This means that the plug region is moving with constant velocity. It's necessary to mention that this strange behavior will fade away in 

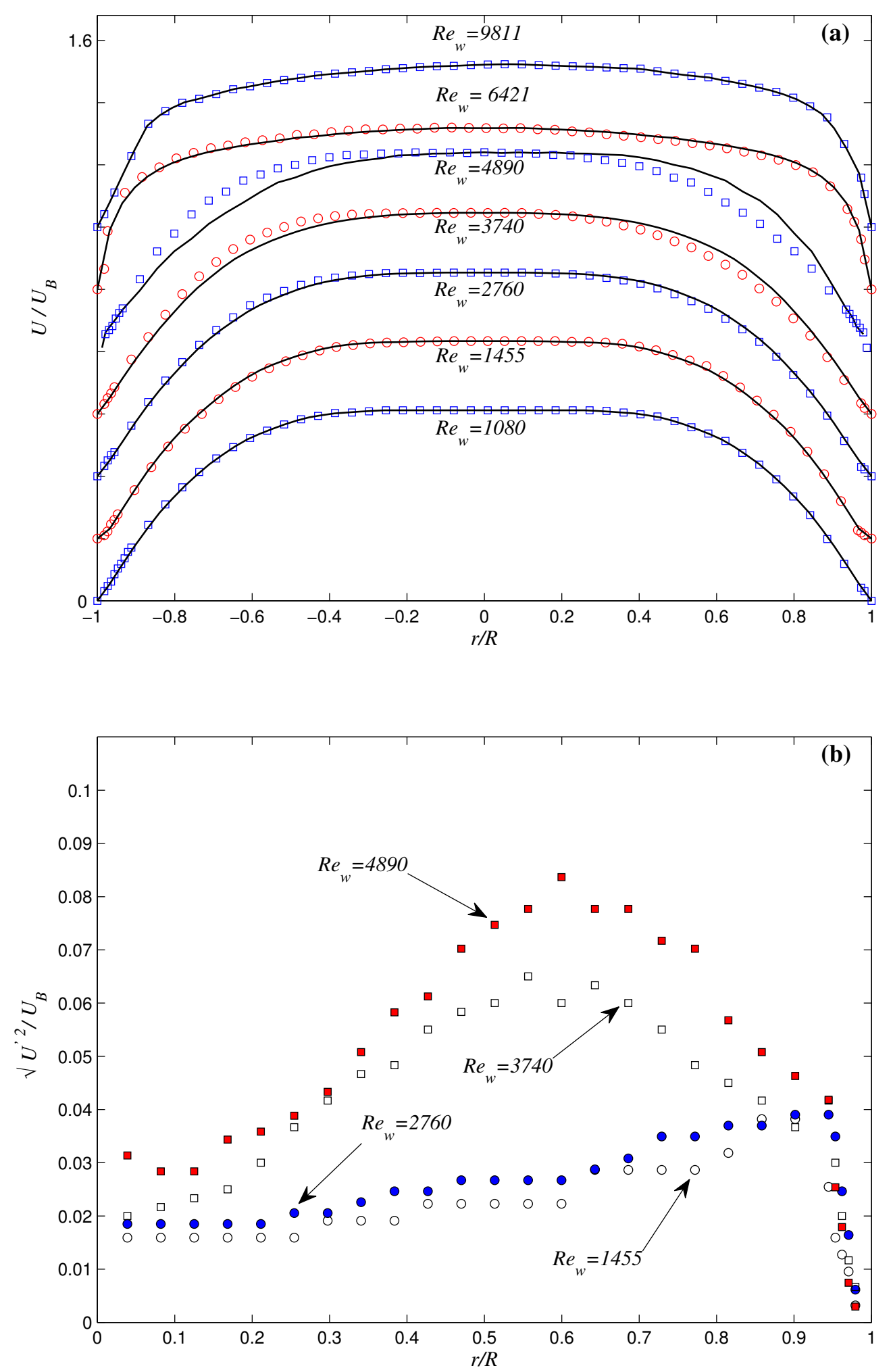

Fig. 2. (a) Mean and (b) fluctuation rate RMS axial velocity profiles of $0.2 \%$ Carbopol at the azimuthal positions of $\theta=0$ and the downstream of the pipe, $\left(\tau_{0}=7.0 \mathrm{~Pa}, \mathrm{~K}=3.1\right.$ Pa.s $\left.^{n}, \mathrm{n}=0.46\right)$. The symbols denote the experimental data and the continuous line is an average velocity profile obtained by taking the average of the velocity data from either side of the centerline. 


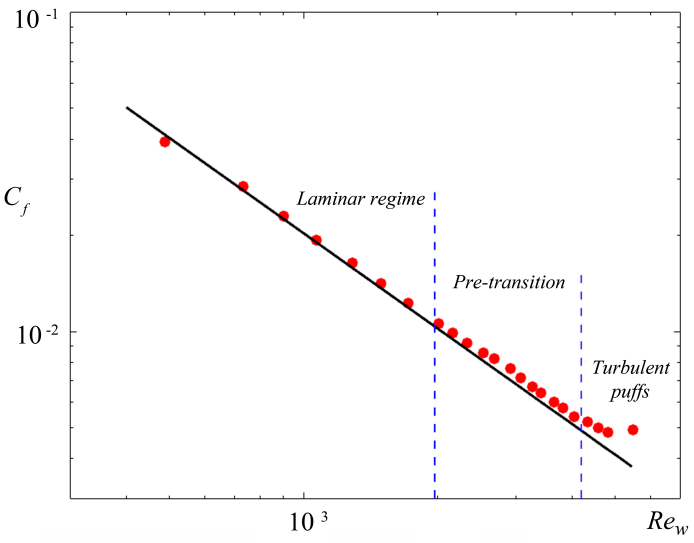

Fig. 3. Friction factor as a function of Reynolds number; The symbols are the experimental measurements and the continuous line is the theoretical laminar solution.

the beginning of a fully developed turbulence. The flow begins to present a stable asymmetry for the Reynolds number corresponding to the transition regime $\left(R e_{w}=2760,3740\right.$ and 4890), while in the laminar and turbulent regimes the flow is axisymmetric. It's worth reminding that, this asymmetry is slight for the pre-transition stage $\left(R e_{w}=2760\right.$ and 3740) and becomes significant for the turbulent puffs stage $\left(R e_{w}=4890\right)$. The similar tendency has also been reported by Güzel et al. (2009); Esmael and Nouar (2008) and Peixinho et al. (2005).

Fluctuation rate axial velocity profiles RMS, corresponding to the mean velocity profiles are shown in Fig. 2b. The profiles are shown normalized using the bulk velocity $\left(U_{B}\right)$. The results show an increase of the fluctuation rate with increasing of $R e_{w}$ outside the plug zone. The profile obtained for $R e_{w}=$ 2760 correspond to the first stage of the transitional regime with a light increase in the fluctuation rate. Thus, the flow can be unstable. This flow instability becomes significant for second stage of transition at $R e_{w}=4890$. The friction factor $C_{f}$ is defined as follows:

$C_{f}=\frac{2 \tau_{p}}{\rho U_{B}^{2}}$

Where the parietal stress $\tau_{p}$ is evaluated from the measurement of $\Delta P$ :

$\tau_{p}=\frac{R}{2} \frac{\Delta P}{L}$

$\rho$ is the density and $\Delta P$ a pressure drop over the length $L$ between the two pressure tappings along the pipe.

The Fig. 3 displays the variation of friction factor $\left(C_{f}\right)$ as a function of Reynolds number. Two different stages are clearly identified during transition

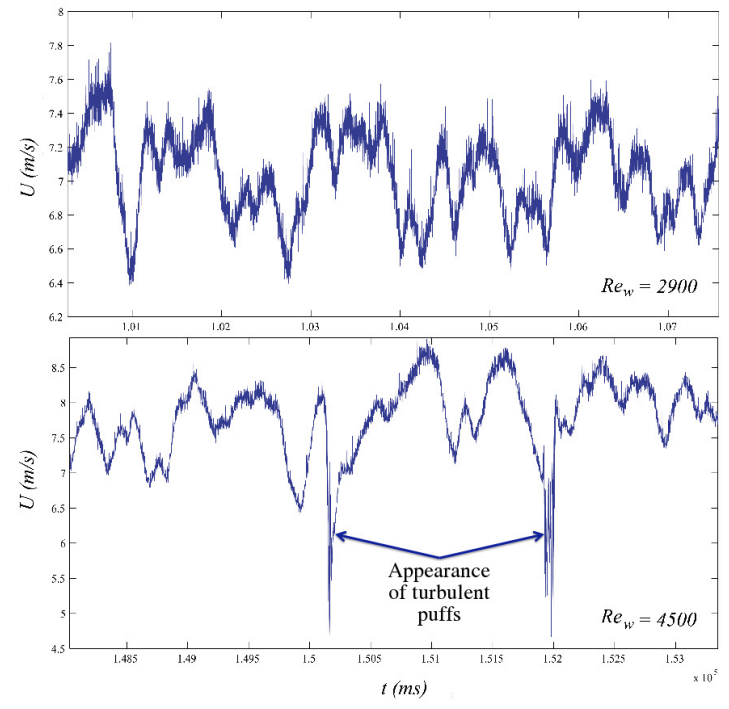

Fig. 4. Instantaneous axial velocity plots at pretransition $R e_{w}=2900$ and turbulent puffs $R e_{w}=$ 4500 transition regime $\left(\theta=0, \frac{r}{R}=0.7\right.$ and the downstream of the pipe).

to turbulence. The first stage, called pre-transition, took place only in the Reynolds number between 2000 and 4000, while the second stage correspond to the appearance of turbulent puffs $\left(R e_{w} i\right.$ 4000). In the pre-transition stage, the experimental measurements of the friction factor are very close to the theoretical laminar solution. We measured axial velocity signals concerning the first stage $\left(R e_{w}=\right.$ $2900)$ and second stage $\left(R e_{w}=4500\right)$ of transitional regime at the $\theta=0, \frac{r}{R}=0.7$ and the downstream of the pipe as shown in Fig. 4. We observed an instantaneous deep fall in velocity at 1.502 and $1.52 \mathrm{~ms}$, which corresponds to the appearance of turbulent puffs at the Reynolds number of 4500.

Elements analysis of these experimental results can be obtained by adopting the Reynolds decomposition for velocity, pressure, shear and effective viscosity. The Reynolds-averaged momentum equation is:

$$
\begin{aligned}
\frac{\partial V_{i}}{\partial t}+\frac{\partial}{\partial x_{k}}\left(V_{i} V_{k}\right)=-\frac{\partial P}{\partial x_{i}} & +\frac{\partial}{\partial x_{k}}\left[\frac{1}{\operatorname{Re}} \overline{\mu^{\prime} \dot{\gamma}_{i k}^{\prime}}-\overline{v_{i}^{\prime} v_{k}^{\prime}}\right] \\
& +\frac{1}{\operatorname{Re}} \frac{\partial}{\partial x_{k}}\left(\bar{\mu} \Gamma_{i k}\right)
\end{aligned}
$$

By comparison with the Newtonian case, there is a new diffusive term $\overline{\mu^{\prime} \dot{\gamma}_{i k}^{\prime}}$, which may be viewed as pseudo Reynolds-stress tensor. The influence of this term on the flow may be analyzed first through the friction factor $C_{f}$. Using an extension of the procedure used in Fukagata et al. (2002), an exact relationship for $C_{f}$ is derived: 


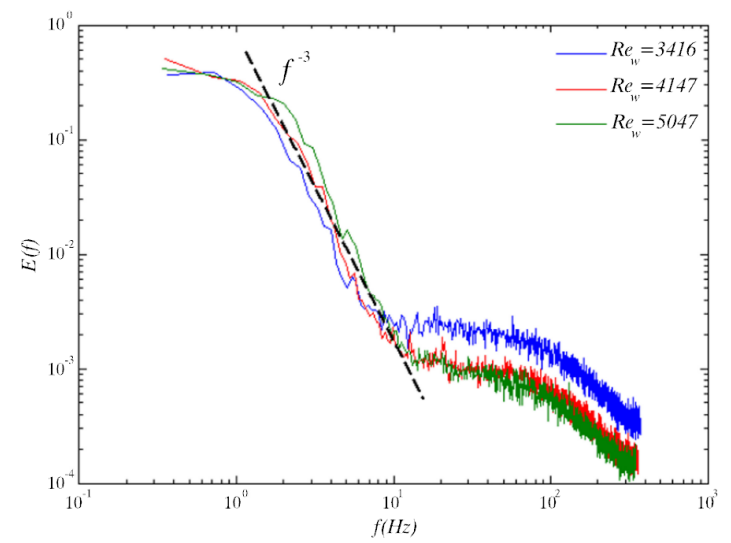

Fig. 5. Density energy spectra of axial velocity fluctuation at the azimuthal positions of $\theta=0, \frac{r}{R}=$ 0.7 and the downstream of the pipe, $\left(\tau_{0}=13.52 \mathrm{~Pa}\right.$,

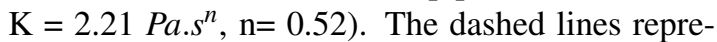
sent the power law behavior $f^{-3}$.

$$
\begin{aligned}
C_{f}=C_{L}+8 \overbrace{8 \int_{0}^{1} r^{2} \overline{v^{\prime} w^{\prime}} d r}^{C_{T}} & -\overbrace{\frac{8}{R e} \int_{0}^{1} r^{2} \overline{\mu^{\prime} \dot{\gamma}_{r z}^{\prime}} d r}^{C_{\mu}^{\prime}} \\
& -\underbrace{\frac{8}{R e} \int_{0}^{1} r^{2} \overline{\mu \Gamma_{r z}} d r}_{C_{M}}
\end{aligned}
$$

This relation shows that the friction factor can be split into four contributing terms: the laminar $C_{L}$, the turbulent contribution $C_{T}$, the viscosity perturbation contribution $C_{\mu^{\prime}}$, and an additional term $C_{M}$ which arises from the modification of the mean velocity profile with respect to the laminar one. It can be shown straightforwardly that for a shearethinning fluid $C_{\mu^{\prime}} i 0$. Thus, this term contributed to be drag reduction and probably compensates the turbulent contribution. This may explain why in this weakly turbulent flow, $C_{f}$ is very close to that in laminar regime.

Power spectra $\mathrm{E}(\mathrm{f})$ of the axial velocity fluctuations at $\frac{r}{R}=0.7$ and horizontal positions $\theta=0$ (near the center of the high speed streak) for the three values of Reynolds, including the first and second stages of transitional regime, are shown in Fig. 5. The density energy spectra were calculated on $5 \times 10^{5}$ data points divided in segments of 2048 points and the obtained spectrum is an average overall segment in the full time series. As seen in Fig. 5, in the transitional regime, the power spectra, identifies the existence of a weak turbulence. It is generated by the shear-thinning character of the fluid, which follows a power law with an exponent close to $-3\left(f^{-3}\right)$ for a decade of $1 \leq f \leq 10 \mathrm{~Hz}$. The flattening of the curves at high frequency is due to an instrumental noise. Recently, Esmael et al. (2010) exhibited an exponent close to $-5 / 3\left(f^{-5 / 3}\right)$ at an early step of transition with increasing $R e_{w}$. The spectra have a broad region of frequencies, where the fluctuation energy decays according to a power law with an exponent close to $-3\left(f^{-3}\right)$. The energy spectra in $f^{-3}$ is very similar to that described in the literature for the case of a two-dimensional turbulence Tabeling (2002).

By adopting the hypothesis of Taylor, the spectra in time can be interpreted by the spectra in space by the relation between the frequency and the wave number given by:

$k=\frac{2 \pi f}{U}$

This can indicate a multiplicity of spatial and temporal scale, which is a fundamental characteristic of a turbulent flow. A steep spectrum $f^{-3}\left(k^{-3}\right)$ means that the energy is mainly concentrated in large-scale structures and the integral scale determines the velocity field. It is thus a weak turbulence which can be assimilated to a Batchelor regim flow Batchelor (1959). In this regim, the flow can be considered smooth in space and random in time and is appears in hydrodynamic turbulence below the dissipation scale Burghelea et al. (2007).

\section{Conclusions}

Two stages are clearly identified during the transition to turbulent. In the first stage called pretransition, a central zone (plug zone) remains in the laminar regime at the fluctuation rate. At this stage the profile of a mean axial velocity exhibits a slight asymmetry and the experimental measurements of the friction factor $C_{f}$ are very close to the theoretical laminar solution, which can be explained by the new diffusive term $\overline{\mu^{\prime} \dot{\gamma}_{i k}^{\prime}}$ as the drag reduction. The second stage corresponds to the appearance of turbulent puffs with a significant asymmetry in the axial velocity profile. In the transitional regime, the power spectra identified the existence of a weak turbulence. This is generated by the shear-thinning character of the fluid, which follows a power law with an exponent close to $-3\left(f^{-3}\right)$.

\section{ACKNOWLEDGMENTS}

The authors are very grateful to Dr. A. Lefèvre and Dr. A. Esmael for fruitful discussions.

\section{REFERENCES}

Ashrafi, N. and R. E. Khayat (2000). Shearthinning-induced chaos in taylor-couette flow. Physical Review E 61, 1455-1467.

Batchelor, G. K. (1959). Small-scale variation of convected quantities like tem- perature in turbulent fluid. Journal of Fluid Mechanics 5, 113-133. 
Burghelea, T., E. Segre, and V. Steinberg (2007). Elastic turbulence in von karman swirling flow between two disks. Physics of Fluids 19, 053104.

Escudier, M. P., F. Presti, and S. Smith (1999). Drag reduction in the turbulent pipe flow of polymers. Journal of Non-Newtonian Fluid Mechanics 81, 197-213.

Escudier, M. P., S. Rosa, and R. J. Poole (2009). Asymmetry in transitional pipe flow of dragreducing polymer solutions. Journal of NonNewtonian Fluid Mechanics 161, 19-29.

Esmael, A. and C. Nouar (2008). Transitional flow of a yield-stress fluid in a pipe: Evidence of a robust coherent structure. Physical Review E 77, 057302.

Esmael, A., C. Nouar, A. Lefèvre, and N. Kabouya (2010). Transitional flow of a non-newtonian fluid in a pipe: Experimental evidence of weak turbulence induced by shear-thinning behavior. Physics of Fluids 22, 101701.

Fukagata, K., K. Iwamoto, and N. Kasagi (2002). Contribution of reynolds stress distribution to the skin friction in wall-bounded.
Physics of Fluids 14, L73-L76.

Groisman, A. and V. Steinberg (2000). Elastic turbulence in a polymer solution flow. $\mathrm{Na}$ ture 405, 53-55.

Güzel, B., T. Burghelea, I. A. Frigaard, and D. M. Martinez (2009). Observation of laminar-turbulent transition of a yield stress fluid in hagen-poiseuille flow. Journal of Fluid Mechanics 627, 97-128.

Larson, R. G. (2000). Turbulence without inertia. Nature 405, 27-28.

Meseguer, A. and L. N. Trefethen (2003). Linearized pipe flow to reynolds number 107 . Journal of Computational Physics 186, 178197.

Peixinho, J., C. Nouar, C. Desaubry, and B. Théron (2005). Laminar transitional and turbulent flow of yield stress fluid in a pipe. Journal of Non-Newtonian Fluid Mechanics 128, 172-184.

Tabeling, P. (2002). Two-dimensional turbulence: a physicist approachs. Physics reports 362, 1-62. 